\title{
Evaluation of Microdissection Testicular Sperm Extraction (mTESE), Outcomes and Predictive Factors in Ireland: The Gold Standard for Men with Non-Obstructive Azoospermia
} \author{
Waterstone ${ }^{2}$, Ivor Cullen ${ }^{1}$ \\ 1- Department of Urology, University Hospital Waterford, Waterford, Ireland \\ 2- Waterstone Clinic, Cork, Ireland \\ 3- College of Science, University College Dublin, Dublin, Ireland \\ 4- Department of Pathology, University Hospital Waterford, Waterford, Ireland
}

Pat Rohan ${ }^{1 *}$, Niamh Daly ${ }^{2}$, Aoife O’Kelly ${ }^{1}$, Martin O’Leary ${ }^{3}$, Tim Dineen ${ }^{2}$, Nigam Shah ${ }^{4}$, Padraig Daly ${ }^{1}$, John

* Corresponding Author: Pat Rohan, 37 Deerpark Road, Castleknock, Dublin, D15 XEA2, Ireland E-mail:

pat.rohan@ucdconnect.ie

Received: Jul. 19, 2020 Accepted: Oct. 20, 2020

\begin{abstract}
Background: Microdissection testicular sperm extraction (mTESE) is the gold standard approach in sperm retrieval in men with non-obstructive azoospermia (NOA). The purpose of the study was to assess the outcomes for Irish men who have undergone mTESE with a single surgeon.

Methods: This is a retrospective, single cohort study. Thirty-four patients underwent mTESE between September 2015 and June 2019. A p $<0.05$ was considered statistically significant.

Results: In this study, sperm retrieval rate (SRR) was $47.06 \%$. (16/34). The mean

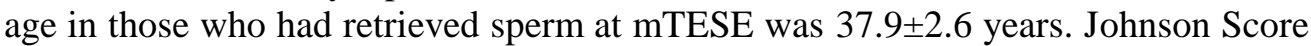
(JS) and FSH were statistically different between successful and unsuccessful mTESE groups $\left(\mathrm{p}=0.017 * 10^{-5}\right.$ and $\mathrm{p}=0.004$, respectively). Optimal cutoff values for FSH, T and JS were $15 \mathrm{IU} / \mathrm{L}, 13 \mathrm{nmol} / \mathrm{L}$ and 5 , respectively. The pregnancy rate was $63.64 \%$ (7/11) among men who went on to use mTESE sperm in an ICSI cycle.

Conclusion: The combination of mTESE/Intracytoplasmic sperm injection (ICSI) is the best option available for men with NOA who prefer to achieve paternity using their own DNA. Given the overall SRRs in mTESE, it is imperative to continue research for a predictive model to better counsel azoospermic men regarding the use of mTESE. For this purpose, large, multicenter, randomized controlled trials are needed.

Keywords: Male infertility, Microdissection testicular sperm extraction, Non-obstructive azoospermia, Predictive markers, Sperm retrieval.

To cite this article: Rohan P, Daly N, O'Kelly A, O'Leary M, Dineen T, Shah N, et al. Evaluation of Microdissection Testicular Sperm Extraction (mTESE), Outcomes and Predictive Factors in Ireland: The Gold Standard for Men with Non-Obstructive Azoospermia. J

Reprod Infertil. 2021;22(2):103-109. http://dx.doi.org/10.18502/jri.v22i2.5795.
\end{abstract}

\section{Introduction}

A zoospermia is defined as the absence of sperm in the ejaculate after two samples have been processed by centrifugation (1). It is seen in $1 \%$ of the male population and in $10-15 \%$ of infertile men (1-3). Non-obstructive azoospermia (NOA) accounts for $60 \%$ and obstructive azoospermia (OA) the remainder. NOA occurs as a result of testicular failure and is divided into two main subtypes of primary (Testicular) NOA and secondary (Pre-testicular) due to endocrine dysfunction of the hypothalamic-pituitary-gonadal (HPG) axis. Endocrine dysfunction of the HPG axis can result in hypogonadotropic hypogonadism (Low follicle stimulating hormone (FSH), luteinising hormone (LH) and testosterone (T)), which can be congenital (Most commonly Kall- 
mann syndrome) or acquired due to tumors or ischaemia. Testicular causes resulting in hypergonadotropic hypogonadism (High FSH and LH and low T) are most often idiopathic. The most commonly known causes are Klinefelter syndrome and $\mathrm{Y}$ chromosome microdeletions in azoospermic factors (AZF) A, B or C $(1,4,5)$.

While azoospermic men have no spermatozoa in the ejaculate, this does not imply sterility as many of these men have ongoing spermatozoa production within the testicle (6). Locating sites of sperm production within the testicle presents a clinical challenge. The seminiferous tubules are responsible for sperm production in the testicle (7). Seminiferous tubules that contain all the cells required for spermatogenesis, namely spermatogonia (Germ cells), appear larger and whiter (But still somewhat yellow) than those tubules where there is no sperm production (8). Sertoli cells have a supportive function within the tubules, facilitating spermatogenesis (9). In contrast, tubules that only contain Sertoli cells, are thin and more translucent (8).

Histopathologically, men with NOA can be divided into three groups: (a) hypospermatogenesis, a reduced number of normal spermatogenic cells; (b) maturation arrest (MA), an absence of the latter stages of spermatogenesis; and (c) Sertoli cell only (SCO), a complete absence of germ cells in the seminiferous tubules (3). The Johnsen score (JS) was developed in 1970 to describe spermatogenesis. Tubules are given a score from 10 to 1 describing the main cell type present and the mean and highest JS in the biopsy are then commonly calculated (10). The score is used to grade the predominant histopathological pattern seen in biopsied testicular tissue (3).

First described in 1994 (11), conventional TESE involves the extraction of rare spermatozoa in NOA men. These blind, non-focused biopsies can result in disruption of the testicular arterial supply and risk total devascularisation and lead to subsequent atrophy of the testicle (8). mTESE involves examining testicular tissue under a microscope to identify blood vessels and areas of sperm production within NOA men. mTESE was first described by Schlegel et al. in 1999 (8) and has become the gold standard technique in testicular sperm retrieval (12). The first reported pregnancy in Ireland using sperm retrieved by mTESE occurred in 2016 (13).

Factors that have been reported to affect the success of sperm retrieval in mTESE include age,
FSH, T, prior varicocele repair (VR) and use of clomiphene citrate (CC) (12, 14-18). Increased age, high FSH and low $\mathrm{T}$ levels are associated with reduced spermatogenesis $(12,15,19,20)$. Varicocele repair has been shown to significantly improve sperm parameters such as sperm count, motility and morphology (17). Exogenous $\mathrm{T}$ replacement causes suppression of the HPG axis resulting in further inhibited spermatogenesis. Clomiphene citrate, a selective oestrogen receptor modulator, has been shown to increase $\mathrm{T}$ levels and spermatogenesis (19).

Couples are heavily invested, emotionally and financially in the outcome of their mTESE procedure $(3,21)$. Previous research highlights the difficulty in identifying prognostic indicators for mTESE and TESE (22-24). The purpose of this study was to assess the experience of men in Ireland who have undergone mTESE in a single center, single surgeon environment. Primary outcome was the sperm retrieval rate and how it was impacted by factors including age, FSH, T, prior varicocele repair, clomiphene citrate use and overall testicular histology. Secondary outcomes included fertilization rates, clinical pregnancy rates and live birth rates.

\section{Methods}

This is a retrospective, single cohort study completed using prospectively collected data from a university hospital and a private fertility institute. Thirty-four patients that underwent mTESE in the period between September 2015 and June 2019 were evaluated.

Azoospermia was confirmed via semen analysis. FSH, pre-procedure serum T, highest JS, and full histopathology results were collected for each patient. For those patients that underwent treatment with $\mathrm{CC}$, their $\mathrm{FSH}$ and $\mathrm{T}$ levels after treatment were used. The patients who underwent mTESE but did not have the available results were excluded. Extra clinical data including history of testicular cancer and presence of varicocele were recorded. The exact cause of NOA in each individual case was not investigated. Data was collected through a search of the hospital laboratory system (Histology), laboratory records from the private fertility clinic, the National Integrated Medical Imaging System (NIMIS), and electronic copies of clinic letters. Men with obstructive azoospermia were excluded. All the men in our cohort had the 46XY karyotypeand none had Y chromosome microdeletions. 
VR was performed for men with a clinical grade 3 varicocele measuring $>3 \mathrm{~mm}$ on ultrasound and for those $<3 \mathrm{~mm}$ that were symptomatic. The use of $\mathrm{CC}$ was indicated in men with at least two morning total $\mathrm{T}$ measurements $<8 \mathrm{nmol} / \mathrm{L}$.

mTESE was performed by a single surgeon in a single center to achieve consistency in the process. Access was via median raphe incision. The tunica vaginalis was opened and the testicle delivered. An equatorial incision was made in the testicle under 20X magnification microscope (Carl Zeiss OPMI 1 FR Pro) to identify vascular supply and avoid iatrogenic injury. Detailed analysis of the testicle was then performed under 40X magnification to identify dilated, more opaque seminiferous tubules.

Next, the pre-warmed multipurpose-handlingmedium-complete (MHM-C) was placed in an ICSI dish. When testicular tissue was excised by the surgeon, it was then immersed in medium in the ICSI dish. The embryologist then took one or two tubules on a pre-sterilized slide and the slide was examined for the presence of sperm. Remaining tissue was placed in a $14 \mathrm{ml}$ round bottom tube containing $10 \mathrm{ml}$ of MHM-C which remained heated until the biopsy was finished.

The embryologist then notified the surgeon if no viable, motile sperm had been observed. At this point, more testicular tissue could be biopsied or the surgeon might move to the contralateral testicle. Once the surgeon was happy that sufficient biopsies were obtained, all the samples were transported in a heated unit to the lab in the fertility clinic.

Once at the clinic, the samples were processed in a biological safety cabinet. The tissue was dissected using insulin needles which were pulled along the tubules to release any sperm. Once all tissue was dissected, the tissue and fluid were placed on a $40 \mu \mathrm{m}$ Falcon cell strainer (Cat no: 352340 ), on a $50 \mathrm{ml}$ centrifuge tube and centrifuged at $250 \mathrm{~g}$ for $5 \mathrm{~min}$.

The sample was resuspended in $0.1-0.2 \mu l$ and analyzed for quantity and quality of sperm. If sperm were observed but none was motile, GM501 SpermMobil (Gynemed, Germany) could be added and the sample re-examined to assess if the sample had viable, motile sperm.

Samples were also analyzed in the hospital histopathology lab and JS were recorded.

Analysis was completed using Student T test, Welch's T test, Mood's Median test, Pearson's
Chi Square test, Fisher's exact test and decision tree analysis.

\begin{abstract}
Results
A total of 34 men underwent mTESE procedures. The overall SRR was 15/34 (44.12\%). The mean age of all patients was $37.2 \pm 1.4$ years. The mean age in those who had retrieved sperm at mTESE was $37.9 \pm 2.6$ years while the mean age for those that did not have sperm retrieval was $36.6 \pm 1.5$ years $(p=0.29)$. The median JS was 3.5. In the successful group, the median was 8 and in the unsuccessful group, median was 3 which shows a statistically significant difference $(\mathrm{p}<$ 0.05 ). The difference in FSH levels was significant $(15.03 \pm 6.54 \mathrm{IU} / \mathrm{L}$; normal range 1.5-12.4 $I U / L)$ in the successful group compared to unsuccessful group $(28.11 \pm 6.26 \mathrm{IU} / \mathrm{L}, \mathrm{p}=0.004)$. Testosterone was not significantly different $(15.08 \pm 2.19$ $\mathrm{nmol} / \mathrm{L}$; normal range 9.9-27.8 $\mathrm{nmol} / \mathrm{L}$ ) in the successful group, compared to unsuccessful group $(16.99 \pm 3.59 \mathrm{nmol} / \mathrm{L}, \mathrm{p}=0.344)$. Varicocele repair did not significantly impact SRR (Pearson's Chi Square $=0.344, p=0.558$ ). CC use did not significantly affect the success of mTESE (Pearson's Chi Square $=1.872, \mathrm{p}=0.171$ ). Table 1 documents the comparison between 6 factors in successful and unsuccessful mTESE.
\end{abstract}

Cutoff values: Decision tree analysis was used to determine potential cutoff values for the highest JS, FSH and T. The proposed FSH cut off value of $15 \mathrm{IU} / \mathrm{L}$ was used. If FSH was $<15 \mathrm{IU} / \mathrm{L}$, the likelihood of sperm retrieval in this cohort was $90 \%$. If FSH was $>=15 \mathrm{IU} / \mathrm{L}$, the likelihood of successful retrieval was $25 \%$. This is in keeping with the significant difference in mean FSH values between the successful and unsuccessful groups. The proposed T cutoff was $13 \mathrm{nmol} / \mathrm{L}$. In our cohort, if $\mathrm{T}$ was $<13 \mathrm{nmol} / \mathrm{L}$, the chance of sperm retrieval was $36 \%$, and if $>=13 \mathrm{nmol} / \mathrm{L}$, the chance of successful sperm retrieval was $60 \%$. With respect to the highest JS, if this value was $<5$, the likelihood of sperm retrieval was $6 \%$, and if $>=5$, the likelihood was $88 \%$.

The number of ICSI cycles that can be undertaken is dependent on the quantity of sperm successfully retrieved. All ICSI cycles used frozen mTESE sperm. In our cohort, the number of cycles undertaken varied from 1 cycle to 4 . Of the 15 men who successfully had retrieved sperm, $4 / 15(26.67 \%)$ had not used the mTESE sperm in an ICSI cycle at the time of analysis and 10/11 
Table 1. Summary of preoperative risk factors

\begin{tabular}{lccccc}
\hline Variables & All patients & $\begin{array}{c}\text { Successful mTESE } \\
(\mathbf{9 5 \%} \mathbf{C I})\end{array}$ & $\begin{array}{c}\text { Unsuccessful mTESE } \\
(\mathbf{9 5 \%} \text { CI) }\end{array}$ & p-value \\
\hline Age & & $37.2 \pm 1.4$ & $37.9 \pm 2.6$ & $36.6 \pm 1.5$ & 0.29 \\
FSH IU/L & $22.34 \pm 4.87$ & $15.03 \pm 6.54$ & $28.11 \pm 6.26$ & 0.004 \\
Testosterone $\boldsymbol{n m o l} / \mathbf{L}$ & & $16.14 \pm 2.14$ & $15.08 \pm 2.19$ & $16.99 \pm 3.59$ & 0.344 \\
Johnsen score (Median) & & 3.5 & 8 & 3 & $<0.05$ \\
Prior varicocele repair \% & & & & & \\
& Yes & $17.65 \%$ & $13.33 \%$ & $21.05 \%$ & 0.558 \\
& No & $82.35 \%$ & $86.67 \%$ & $78.95 \%$ & \\
Clomiphene use \% & & & & & \\
& Yes & $32.35 \%$ & $20 \%$ & $52.11 \%$ & 0.171 \\
& No & $67.65 \%$ & $80 \%$ & $57.89 \%$ & \\
\hline
\end{tabular}

Table 2. Summary of clinical outcomes

\begin{tabular}{lccc}
\hline Outcome & Yes & No & \% \\
\hline Fertilization & 10 & 1 & $90.91 \%$ \\
Pregnancy & 7 & 4 & $63.64 \%$ \\
Live birth & 5 & 6 & $45.45 \%$ \\
\hline
\end{tabular}

(90.91\%) men who had mTESE sperm used in an ICSI cycle had at least successful fertilization from the mTESE sperm. Moreover, 7/11 (63.64\%) went on to have at least a pregnancy result from the mTESE sperm and 5/11 (45.45\%) went on to have a live birth resulting from sperm retrieved with mTESE. Of note, 3 men had separate mTESE/ICSI cycles resulting in pregnancy that did not proceed to a live birth, as well as an mTESE/ICSI cycle that did proceed to a live birth. The results are summarized in table 2 .

\section{Discussion}

Microdissection testicular sperm extraction (mTESE) is the gold standard for testicular sperm retrieval (12) and in combination with intracytoplasmic sperm injection (ICSI), men who were previously considered unable to father children may now do so (2). While there are multiple options available for retrieving sperm in men with NOA, mTESE has become the eminent technique since its inception in $1999(8,25)$. Our data examined the SRR seen in our cohort and analyzed potential predictive factors for the success of mTESE. This is the first paper of its kind in Ireland.

In this cohort, the mTESE SRR was $44.12 \%$ compared to 42.9-63\% reported by Deruyver et al. in their systematic review (25). Their reported
SRR was $16.7-45 \%$ in conventional TESE (25). Ishikawa also demonstrated consistently higher SRRs in mTESE over conventional TESE (20). Along with improved SRRs, mTESE is associated with a more favorable complication profile (5). It is proposed that mTESE allows for easier identification of testicular blood vessels and is associated with limited tissue excision resulting in less testicular damage (15).

There is a lack of prospective randomized controlled trials comparing mTESE with other sperm retrieval techniques but a systematic review comparing conventional TESE with mTESE demonstrated significantly higher sperm retrieval rates (SRRs) in mTESE (42.9-63\% compared to 16.7$45 \%)(25)$.

In our analysis, the mean FSH in those with successful sperm retrieval was $15.03 \pm 6.54 \mathrm{IU} / \mathrm{L}$, whereas in the unsuccessful cohort, the mean was $28.1 \pm 6.26 \mathrm{IU} / \mathrm{L}$, which showed a significant difference ( $\mathrm{p}=0.004)$. This result adds to the ambiguity surrounding FSH as a predictive marker.

Multiple studies to date promote the use of FSH as a predictive marker for successful mTESE (24, 26), while others suggest serum FSH concentration does not impact SRR $(15,27)$. Serum FSH is inversely proportional to the number of germ cells present and therefore elevated FSH levels have been associated with an absence of spermatozoa. FSH level may reflect the predominant pattern of spermatogenesis but it does not reflect the presence of isolated areas of spermatogenesis or the stage of spermatogenesis in these areas $(12,15$, 20). As a result, excellent SRRs may be seen in those with elevated FSH $(12,27)$.

A proposed cutoff value for FSH was $15 \mathrm{IU} / \mathrm{L}$, 
the same as that used by Cetinkaya et al. (24). They showed a sensitivity of $75 \%$ and specificity of $51.2 \%$ when this cutoff value was used. In our cohort, $90 \%$ of those with FSH $<15$ IU/L had successful sperm retrieval.

The greatest JS of testicular tissue was taken during mTESE. In men who had the successful mTESE, the mean score was 7.8, compared to 2.84 in men with unsuccessful sperm retrieval which is a significant difference $(p<0.05)$. The optimal cutoff score of 5 resulted in a $6 \%$ chance of sperm retrieval if JS was $<5$ and $88 \%$ if $>=5$. In contrast, Cetinkaya et al. chose a JS cutoff value of 2 (24).

In our data, there was no significant difference in $T$ levels amongst those who had successful mTESE compared with those that did not $(\mathrm{p}=0.344)$. However, an optimal cutoff level for $\mathrm{T}$ was examined in this study and this was found to be $13 \mathrm{nmol} / \mathrm{L}$. $\mathrm{CC}$ use to improve $\mathrm{T}$ levels did not significantly affect the success of mTESE in our patients. There is a mixed picture in the literature regarding testosterone levels and SRR. Various papers have found $\mathrm{T}$ levels to be significant predictive markers $(15,24)$, while others have found no significance in rates of successful sperm retrieval (26).

In men with NOA and hypogonadism, testosterone deficiency is a result of failure along the HPG axis. Treating hypogonadism with exogenous testosterone results in suppression of LH and FSH secretion, thereby causing impaired spermatogenesis (19). CC has been used as an effective method of increasing gonadotrophin levels and consequently testosterone and sperm production (19).

Histological subtype, karyotype and Y chromosome microdeletions are known to affect SRR (12). SRRs vary significantly according to histological subtype (20). Hypospermatogenesis has the highest SRR at $73-100 \%$, while maturation arrest and SCO have SRRs of $27-86 \%$ and 27$40 \%$, respectively (12). Prior invasive testicular biopsy is required in order to determine the histological subtype but this is not ideal given the potential for adverse effects on potentially viable testes, including devascularization, fibrosis and atrophy $(5,15,20,28)$.

Karyotyping and $\mathrm{Y}$ chromosome microdeletions also provide very useful information regarding the likelihood of successful mTESE but abnormalities are seen in the minority of men with NOA (24). All patients included here had 46XY karyotype and none had Y chromosome microdeletions.
Serum inhibin B was not measured or analyzed in our cohort but has been suggested by some as a predictive marker for successful sperm retrieval in NOA, in isolation or in combination with other markers $(14,24,26)$. Others ,however, have found it does not indicate the presence of spermatozoa $(15,29-31)$. The physiology of inhibin B production in the adult is controversial. There is a negative feedback loop between inhibin B and FSH, and while it is an indicator of spermatogenesis, it is not known exactly how germ cells influence inhibin B production by sertoli cells. Thus, its value in individual cases remains contentious (32).

In this cohort, varicocele repair did not significantly impact SRR. Varicocele repair may result in presence of sperm in the ejaculate in $10 \%$ of men who previously had no detectable sperm, 3-6 months after the repair. This is more likely in those with hypospermatogenesis and late maturation arrest compared to sertoli cell only (SCO) (12). There is conflicting evidence on whether varicocele repair improves SRRs (12).

Tsujimura has suggested two models for predicting successful sperm retrieval (15). These predictive models were generated by multivariate logistic regression analysis. The first model includes only non-invasive markers including $\mathrm{FSH}, \mathrm{T}$ and inhibin B with sensitivity of $71 \%$ and specificity of $71.4 \%$. The predicted probability of successful mTESE was defined as $\mathrm{p}=[1+\exp (5.201-(0.048 \times$ serum FSH $)-(0.449 \times$ total $\mathrm{T})-(0.021 \times$ serum inhibin $\mathrm{B})]^{-1}(14)$.

The second model includes histological JS (Taken at the time of mTESE as opposed to from a preoperative diagnostic biopsy) as well as age and FSH for prediction (15). The predicted probability is calculated by $\mathrm{p}=[1+\exp ((0.144 \times$ patient age $)$ $-(0.059 \times$ serum FSH $)-(1.310 \times \mathrm{JS}))]^{-1}(14)$. The sensitivity and specificity were $78 \%$ and $76.3 \%$, respectively. It seems that no prospective, well designed, randomized trials have been carried out to test these models.

Multiphoton microscopy (MPM) is a developing technology with applications in multiple areas including neuroscience, oncology and urology (33). With respect to NOA, MPM can potentially be used to identify seminiferous tubules with active spermatogenesis in real time (33). In a rodent model, Ramasamy et al. were able to identify the stage of spermatogenesis within tubules and to identify the presence or absence of sperm (34). Another study examining testicular biopsy sam- 
ples, showed correlations in diagnoses using MPM to traditional hematoxylin and eosin staining (35). Further investigation is needed into the potential side effects of MPM lasers before this technology is expanded for use in humans (33).

The mTESE/ICSI combination is the best option available for men with NOA who prefer to father their own children when no sperm is available in the ejaculate. The physical, psychological, and financial consequences can be extremely difficult for patients and the development of a set of markers that can reliably predict outcome of MTESE would greatly alleviate these patients' stresses $(15,24)$.

Given the less than ideal SRRs in mTESE, it is imperative to continue the search for a predictive model in an attempt to alleviate all the stresses associated with unsuccessful mTESE. For this purpose, large, multicenter, randomized controlled trials are needed along with the development of new technologies.

\section{Conclusion}

This paper confirms the excellent SRRs seen in mTESE. Our data suggests FSH and JS may be used to help predict successful outcome, but robust experimental study designs are needed to prove the correlation. This data confirms that sperm retrieved using mTESE results in a high rate of pregnancy. The mTESE/ICSI process has provided 5 live births for couples in Ireland who otherwise may not have been able to have their own children.

\section{Conflict of Interest}

There are no conflicts of interest.

\section{References}

1. Dabaja AA, Schlegel PN. Microdissection testicular sperm extraction: an update. Asian J Androl. 2013; 15(1):35-9.

2. Bernie AM, Mata DA, Ramasamy R, Schlegel PN. Comparison of microdissection testicular sperm extraction, conventional testicular sperm extraction, and testicular sperm aspiration for nonobstructive azoospermia: a systematic review and meta-analysis. Fertil Steril. 2015;104(5):1099-103.e1-3.

3. Abdel Raheem A, Garaffa G, Rushwan N, De Luca F, Zacharakis E, Abdel Raheem T, et al. Testicular histopathology as a predictor of a positive sperm retrieval in men with non-obstructive azoospermia. BJU Int. 2013;111(3):492-9.

4. Aziz N, Agarwal A. The diagnosis and treatment of male infertility: a case-based guide for clinicians. 1st ed. USA: Springer; 2017. 318 p.

5. Donoso P, Tournaye H, Devroey P. Which is the best sperm retrieval technique for non-obstructive azoospermia? a systematic review. Hum Reprod Update. 2007;13(6):539-49.

6. Esteves SC, Miyaoka R, Agarwal A. An update on the clinical assessment of the infertile male. Clinics (Sao Paulo). 2011;66(4):691-700.

7. Johnson L. Evaluation of the human testis and its age-related dysfunction. Prog Clin Biol Res. 1989; 302:35-60.

8. Schlegel PN. Testicular sperm extraction: microdissection improves sperm yield with minimal tissue excision. 1999;14(1):131-5.

9. Griswold MD. The central role of sertoli cells in spermatogenesis. Semin Cell Dev Biol. 1998;9(4): 411-6.

10. Johnsen SG. Testicular biopsy score count--a method for registration of spermatogenesis in human testes: normal values and results in 335 hypogonadal males. Hormes. 1970;1(1):2-25.

11. Devroey P, Liu J, Nagy Z, Tournaye H, Silber SJ, Van Steirteghem AC. Normal fertilization of human oocytes after testicular sperm extraction and intracytoplasmic sperm injection. Fertil Steril. 1994;62(3):639-41.

12. Flannigan R, Bach PV, Schlegel PN. Microdissection testicular sperm extraction. Transl Androl Urol. 2017;6(4):745-52.

13. Waterstone Clinic. First Pregnancy following Micro-TESE in Ireland [Internet]. Waterstone Clinic: Ireland; 1 Jun 2016. Available from: https:// waterstoneclinic.ie/first-micro-tese-pregnancyireland/

14. Tsujimura A, Matsumiya K, Miyagawa Y, Takao T, Fujita K, Koga M, et al. Prediction of successful outcome of microdissection testicular sperm extraction in men with idiopathic nonobstructive azoospermia. J Urol. 2004;172(5 Pt 1):1944-7.

15. Tsujimura A. Microdissection testicular sperm extraction: Prediction, outcome, and complications. Int J Urol. 2007;14(10):883-9.

16. Schlegel PN, Tanrikut C, Li PS. Microdissection testicular sperm extraction (TESE) in non-obstructive azoospermia. Fertil Steril. 2006;86(3): S519.

17. Agarwal A, Deepinder F, Cocuzza M, Agarwal R, Short RA, Sabanegh E, et al. Efficacy of varicocelectomy in improving semen parameters: new meta-analytical approach. Urology. 2007;70(3): 532-8. 
18. Dadhich P, Ramasamy R, Scovell J, Wilken N, Lipshultz L. Testosterone versus clomiphene citrate in managing symptoms of hypogonadism in men. Indian J Urol. 2017;33(3):236-40.

19. Katz DJ, Nabulsi O, Tal R, Mulhall JP. Outcomes of clomiphene citrate treatment in young hypogonadal men. BJU Int. 2012;110(4):573-8.

20. Ishikawa T. Surgical recovery of sperm in nonobstructive azoospermia. Asian J Androl. 2012;14 (1):109-15.

21. Crabbe E, Verheyen G, Silber S, Tournaye H, Van de Velde A, Goossens A, et al. Enzymatic digestion of testicular tissue may rescue the intracytoplasmic sperm injection cycle in some patients with non-obstructive azoospermia. Hum Reprod. 1998;13(10):2791-6.

22. Tournaye H, Verheyen G, Nagy P, Ubaldi F, Goossens A, Silber S, et al. Are there any predictive factors for successful testicular sperm recovery in azoospermic patients? Hum Reprod. 1997;12(1): 80-6.

23. Eken A, Gulec F. Microdissection testicular sperm extraction (micro-TESE): Predictive value of preoperative hormonal levels and pathology in nonobstructive azoospermia. Kaohsiung J Med Sci. 2018;34(2):103-8.

24. Cetinkaya M, Onem K, Zorba OU, Ozkara H, Alici B. Evaluation of microdissection testicular sperm extraction results in patients with non-obstructive azoospermia: independent predictive factors and best cutoff values for sperm retrieval. Urol J. 2015; 12(6):2436-43.

25. Deruyver Y, Vanderschueren D, Van der Aa F. Outcome of microdissection TESE compared with conventional TESE in non-obstructive azoospermia: a systematic review. Andrology. 2014;2(1): 20-4.

26. Ziaee SA, Ezzatnegad M, Nowroozi M, Jamshidian $\mathrm{H}$, Abdi H, Moghaddam SMMH. Prediction of successful sperm retrieval in patients with nonobstructive azoospermia. Urol J. 2006;3(2):92-6.
27. Ramasamy R, Lin K, Gosden LV, Rosenwaks Z, Palermo GD, Schlegel PN. High serum FSH levels in men with nonobstructive azoospermia does not affect success of microdissection testicular sperm extraction. Fertil Steril. 2009;92(2):590-3.

28. Ramasamy R, Yagan N, Schlegel PN. Structural and functional changes to the testis after conventional versus microdissection testicular sperm extraction. Urology. 2005;65(6):1190-4.

29. Tunc L, Kirac M, Gurocak S, Yucel A, Kupeli B, Alkibay T, et al. Can serum Inhibin B and FSH levels, testicular histology and volume predict the outcome of testicular sperm extraction in patients with non-obstructive azoospermia? Int Urol Nephrol. 2006;38(3-4):629-35.

30. Jensen CFS, Ohl DA, Hiner MR, Fode M, Shah T, Smith GD, et al. Multiple needle-pass percutaneous testicular sperm aspiration as first-line treatment in azoospermic men. Andrology. 2016;4(2): 257-62.

31. Vernaeve V, Tournaye H, Schiettecatte J, Verheyen G, Steirteghem AV, Devroey P. Serum inhibin $\mathrm{B}$ cannot predict testicular sperm retrieval in patients with non-obstructive azoospermia. Hum Reprod. 2002;17(4):971-6.

32. Meachem SJ, Nieschlag E, Simoni M. Inhibin B in male reproduction: pathophysiology and clinical relevance. Eur J Endocrinol. 2001;145(5):561-71.

33. Katz MJ, Huland DM, Ramasamy R. Multiphoton microscopy: applications in Urology and Andrology. Transl Androl Urol. 2014;3(1):77-83.

34. Ramasamy R, Sterling J, Fisher ES, Li PS, Jain M, Robinson BD, et al. Identification of Spermatogenesis With Multiphoton Microscopy: An Evaluation in a Rodent Model. J Urol. 2011;86(6):248792.

35. Najari BB, Ramasamy R, Sterling J, Aggarwal A, Sheth S, Li PS, et al. Pilot study of the correlation of multiphoton tomography of ex vivo human testis with histology. J Urol. 2012;188(2):538-43. 